\title{
A Simple, Safe, and Environmentally Friendly Method of FaSSIF and FeSSIF Preparation Without Methylene Chloride
}

\author{
Hitesh Jogia*, Surya Prakash Sola, Lovleen Kumar Garg, Srinivas Arutla, \\ Annarapu Malleswara Reddy, and Vobalaboina Venkateswarlu \\ Integrated Product Development, Dr. Reddy's Laboratories Ltd, Bachupally, Hyderabad-500090, India
}

\begin{abstract}
Biorelevant dissolution media were designed and proposed based on physiological and physicochemical properties of the small intestine luminal contents in the fasted- and fed-state conditions. Biorelevant dissolution media have proved to be an excellent in vitro tool for predicting the in vivo performance of formulations in fasted-and fed-state conditions. Taurocholic acid and lecithin are surfactants used in biorelevant dissolution media to solubilize the drugs in dissolution media, which mimics the solubilization process of drugs in vivo.

Preparation of biorelevant dissolution media is tedious and complex; it involves the emulsification of lecithin with methylene chloride followed by evaporation of methylene chloride under vacuum. In this work, an attempt was made to simplify biorelevant media preparation by applying high-speed stirring without methylene chloride. Biorelevant dissolution media prepared by the stirring method were clear and transparent. The performance of the media was compared by a dissolution study of two formulations in FaSSIF and FeSSIF media prepared by both the proposed method and a conventional method. The results of the dissolution test were comparable. The proposed method of biorelevant dissolution media preparation is simple, safe, reproducible, and less time consuming.
\end{abstract}

KEYWORDS: FaSSIF, FeSSIF, environmentally friendly, dissolution, surfactants, biorelevant media.

\section{INTRODUCTION}

$\mathrm{P}$ redicting the in vivo performance of a formulation is an ambitious target for formulators. In vitro dissolution testing is one of the best tools for predicting the in vivo performance of drugs. Simulated small intestinal biorelevant media are increasingly seen as a helpful tool to assess the dissolution and solubility of drugs. Fasted-state simulated intestinal fluids (FaSSIF) and fed-state simulated intestinal fluids (FeSSIF), which contain the natural solubilizers bile salt and lecithin in amounts similar to intestinal fluids, were introduced by Dressman (1). The biorelevant dissolution media have proved to be an excellent tool for predicting the in vivo performance of a formulation. Biorelevant media are also of interest to anticipate potential food effects and to model drug-absorption processes (2).

FeSSIF and FaSSIF contain natural surfactants that form more complex lipid aggregates. Methods of preparing biorelevant media involve emulsification in methylene chloride (3) or sequential addition (4). The conventional preparation methods for FeSSIF and FaSSIF are timeconsuming and require organic solvents; residual organic solvents, if present in media, may affect physicochemical properties and dissolution behavior (5).

In this work, the preparation of FaSSIF and FeSSIF without the use methylene chloride was investigated. Sodium taurochloate was dissolved in blank FaSSIF and FeSSIF

${ }^{*}$ Corresponding author. media, lecithin was added gradually with optimized stirring speed, and finally media were diluted to suitable volume. FaSSIF and FeSSIF media were prepared by both the conventional and proposed methods, and dissolution behavior of these media were compared using the similarity factor $f_{2}$.

\section{MATERIALS AND METHODS}

To prepare media by the conventional and stirring methods, pure phosphatidylcholine (PC) from egg and pure sodium taurocholate (NaTC) were used. PC with a purity of $98.6 \%$ was purchased from Lipoid, Germany. NaTC with a purity of $96.1 \%$ was purchased from New Zealand Pharmaceutical. Acetonitrile, methanol, methylene chloride, monosodium phosphate, sodium acetate, and sodium hydroxide were of analytical grade. The stirrer was purchased from Remi Motors Ltd., India (model ROT-124A).

\section{Drugs and Dosage Forms}

Metaxalone tablets $(800 \mathrm{mg}$ ) were provided by $\mathrm{Dr}$ Reddy's laboratory (Hyderabad, India). Metaxalone is a weakly basic BCS Class 2 drug.

Dexlansoprazole capsules $(60 \mathrm{mg}$ ) that contained a mixture of delayed- and extended-release pellets were provided by Dr Reddy's laboratory (Hyderabad, India).

\section{Dissolution Media Preparation}

Conventional FaSSIF and FeSSIF were prepared as per the literature (3). 


\section{Preparation of Blank FaSSIF pH 6.5}

Sodium hydroxide pellets $(1.74 \mathrm{~g}), 19.77 \mathrm{~g}$ of sodium dihydrogen phosphate monohydrate or $17.19 \mathrm{~g}$ of anhydrous sodium dihydrogen phosphate, and $30.93 \mathrm{~g}$ of sodium chloride were dissolved in $5 \mathrm{~L}$ of purified water. The $\mathrm{pH}$ was adjusted to exactly 6.5 using $1 \mathrm{~N}$ sodium hydroxide or $1 \mathrm{~N} \mathrm{HCl}$.

\section{Preparation of FaSSIF pH 6.5}

Sodium taurocholate $(16.5 \mathrm{~g})$ was dissolved in $2000 \mathrm{~mL}$ of blank FaSSIF buffer solution. Lecithin $(6.1 \mathrm{~g})$ was added slowly with vigorous stirring by a homogenizer at about $4000 \mathrm{rpm}$ for approximately $30 \mathrm{~min}$ or until the solution became clear. After the solution became clear, it was diluted to $10 \mathrm{~L}$ with blank FaSSIF.

\section{Preparation of Blank FeSSIF pH 5.0}

Sodium hydroxide pellets $(44 \mathrm{~g})$ dissolved in $87 \mathrm{~mL}$ of glacial acetic acid and $118.8 \mathrm{~g}$ of sodium chloride were added to $10 \mathrm{~L}$ of purified water. The $\mathrm{pH}$ was adjusted to exactly 5.0 using $1 \mathrm{~N}$ sodium hydroxide or $1 \mathrm{~N} \mathrm{HCl}$.

\section{Preparation of FeSSIF pH 5.0}

Sodium taurocholate $(82.5 \mathrm{~g}$ ) was dissolved in $2000 \mathrm{~mL}$ of blank FaSSIF buffer solution, and then $29.5 \mathrm{~g}$ of lecithin was added slowly with vigorous stirring by a homogenizer at about $4000 \mathrm{rpm}$ for approximately $45 \mathrm{~min}$ or until the solution became clear. After the solution became clear, it was diluted to $10 \mathrm{~L}$ with blank FeSSIF.

\section{Dissolution Test}

Metaxalone Tablets $800 \mathrm{mg}$

The dissolution studies were performed with USP Apparatus 2 (paddle) (Lablndia, India) employing 900 $\mathrm{mL}$ of dissolution medium (FeSSIF) at $37 \pm 0.5^{\circ} \mathrm{C}$ and a stirring rate of $50 \mathrm{rpm}$. A sample of approximately $10 \mathrm{~mL}$ was removed from each vessel using an auto sampler and was replaced immediately with approximately 10 $\mathrm{mL}$ of fresh medium at $37 \pm 0.5^{\circ} \mathrm{C}$. The samples were filtered through $10-\mu \mathrm{m}$ filters, and $5 \mathrm{~mL}$ was diluted to $10 \mathrm{~mL}$ with a solution of water/methanol $(55 / 50, \mathrm{v} / \mathrm{v})$ and assayed by HPLC. The dissolution test was performed on six units $(n=6)$.

\section{Dexlansoprazole Capsules $60 \mathrm{mg}$}

The dissolution studies were performed with USP Apparatus 2 (paddle) (Lablndia, India) employing $500 \mathrm{~mL}$ of dissolution medium (FaSSIF) at $37 \pm 0.5^{\circ} \mathrm{C}$ and a stirring rate of $50 \mathrm{rpm}$. A sample of approximately $10 \mathrm{~mL}$ was removed from each vessel using an auto sampler and was replaced immediately with approximately $10 \mathrm{~mL}$ of fresh medium at $37 \pm 0.5^{\circ} \mathrm{C}$. The samples were filtered through $10-\mu \mathrm{m}$ filters, and $5 \mathrm{~mL}$ was diluted to $7 \mathrm{~mL}$ with a solution of $0.25 \mathrm{~N}$ sodium hydroxide and assayed by HPLC. The dissolution test was performed on six units $(n=6)$.

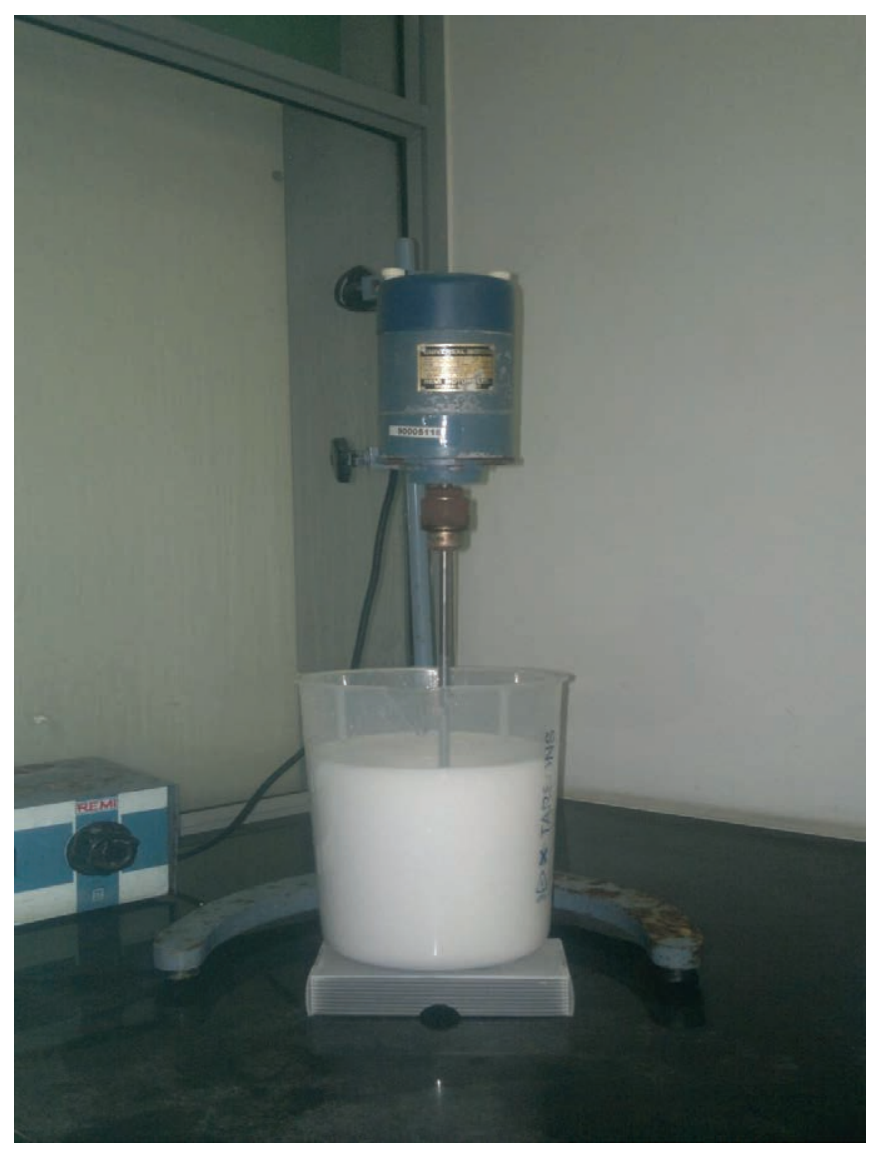

Figure 1. Addition of taurocholic acid to blank FaSSIF in the preparation of biorelevant dissolution media without use of methylene chloride (DCM).

\section{Assay Methods}

The assay of metaxalone tablets was performed by HPLC using an X-terra RP8 column $(150 \times 4.6 \mathrm{~mm}, 5-\mu \mathrm{m}$ particle size). The mobile phase consisted of $0.01 \mathrm{M}$ potassium dihydrogen phosphate $(\mathrm{pH} 2.5)$ /acetonitrile $(55 / 45$, $\mathrm{v} / \mathrm{v})$. The flow rate was $1.0 \mathrm{~mL} / \mathrm{min}$. The effluent was monitored at $272 \mathrm{~nm}$ for metaxalone. The retention time of the metaxalone peak was about 3 min.

The assay of dexlansoprazole was performed by HPLC using an X-bridge $\mathrm{C}-18$ column $(20 \times 4.6 \mathrm{~mm}, 5-\mu \mathrm{m}$ particle size). The mobile phases consisted of $0.025 \mathrm{M}$ potassium dihydrogen phosphate $(\mathrm{pH} 8.0) / a c e t o n i t r i l e$ $(90 / 10, v / v)$ and acetonitrile/methanol (50/50, v/v) in a gradient elution program. The flow rate was $1.2 \mathrm{~mL} / \mathrm{min}$. The effluent was monitored at $285 \mathrm{~nm}$ for dexlansoprazole. The retention time of the dexlansoprazole peak was about $3 \mathrm{~min}$.

\section{RESULTS AND DISCUSSION}

Biorelevant dissolution media are useful for predicting the in vivo dissolution of drugs. The preparation methods for FaSSIF and FeSSIF are complex and require emulsification of lecithin in methylene chloride and subsequent solvent evaporation. Residual methylene 


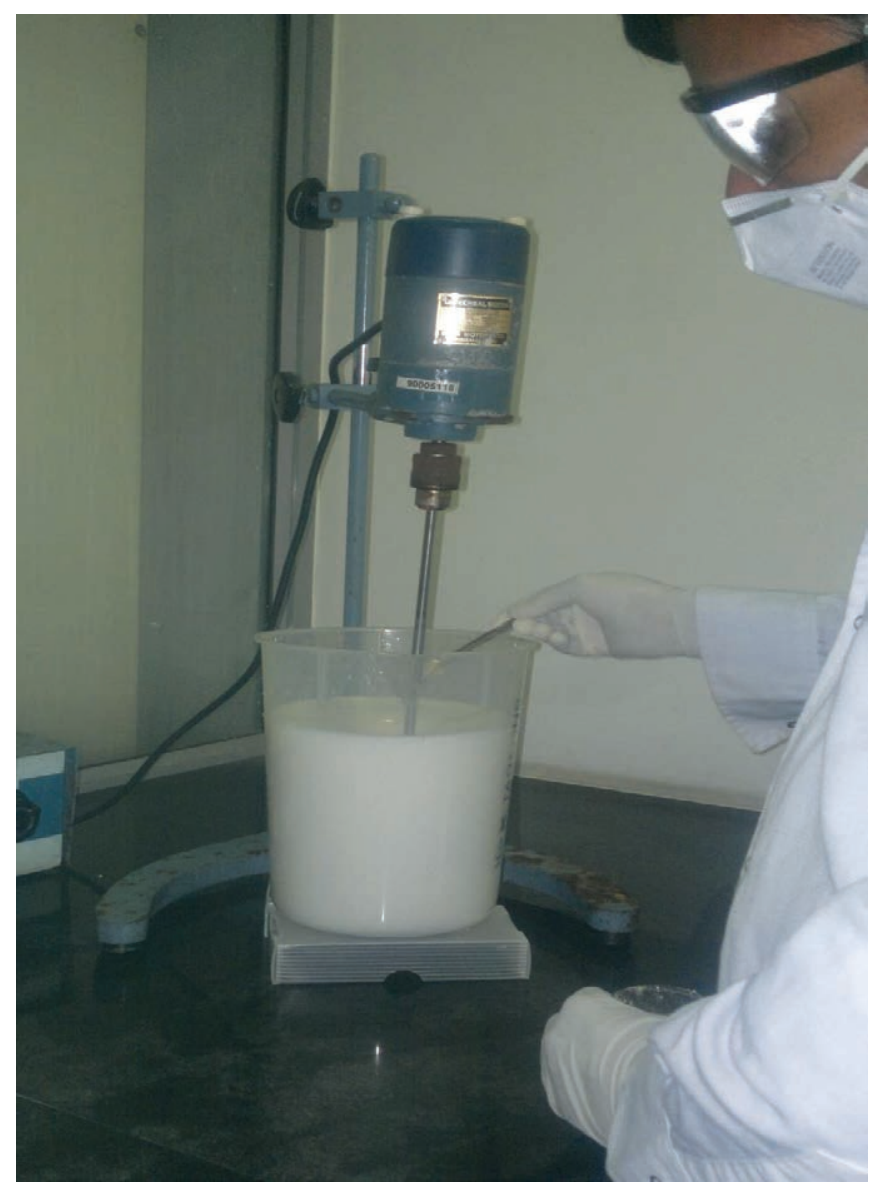

Figure 2. Step-by-step addition of lecithin in the preparation of biorelevant dissolution media without the use of methylene chloride (DCM).

chloride, if present in the dissolution media, may lead to variation in drug dissolution results. To avoid the complexity of FaSSIF and FeSSIF media preparation, lyophilized Simulated Intestinal Fluid (SIF) powder is used for dissolution media preparation (6). For various reasons, SIF powder is not readily available, so alternative methods that are less time-consuming and more environmentally friendly than the original preparation method would be useful and would make biorelevant media available to everyone. To simplify the preparation of FaSSIF and FeSSIF, various approaches were used, such as sequential addition of taurocholic acid and lecithin with stirring. Taurocholic acid (hydrophilic) was dissolved in blank FaSSIF and FeSSIF, then the solution was stirred by means of a homogenizer at about $4000 \mathrm{rpm}$ (Figure 1), and lecithin (lipophilic) was slowly added with stirring to form mixed micelles (Figure 2). A clear micellar solution was obtained (Figure 3).

To evaluate the performance of the biorelevant dissolution media prepared by this simplified approach, dissolution profiles of two formulations were compared using media prepared with methylene chloride (MDC) and without methylene chloride.

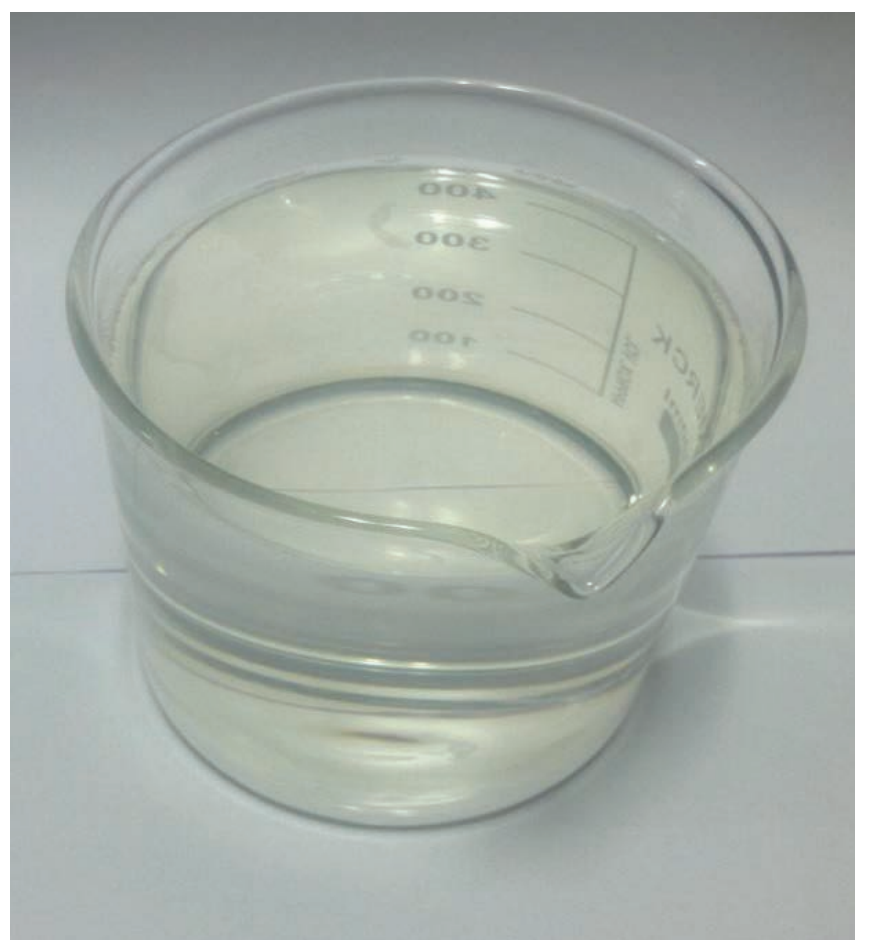

Figure 3. Clarity of FaSSIF medium prepared by the stirring method.

Metaxalone is a BCS Class 2 drug; 800 -mg tablets were used for the dissolution profile comparison in FeSSIF. Figure 4 represents a comparison of the mean in vitro dissolution profiles of metaxalone tablets in FeSSIF media (with and without methylene chloride); the similarity factor $f_{2}$ value obtained was 70 . Reproducibility of the proposed dissolution media was checked by performing in vitro dissolution of 800-mg metaxalone tablets in dissolution media prepared on different days. Figure 5 shows the mean in vitro dissolution profiles of metaxalone tablets. The similarity factor $f_{2}$ value obtained was 77 .

Dexlansoprazole capsules $(60 \mathrm{mg})$ that contained delayed-release and extended-release pellets were used to evaluate FaSSIF dissolution media. Figure 6 shows a comparison of the mean in vitro dissolution profiles of 60$\mathrm{mg}$ dexlansoprazole capsules in FaSSIF media (with and without methylene chloride); the similarity factor $f_{2}$ value obtained was 78.

The dissolution profiles of both products in FaSSIF and FeSSIF were similar to those in media prepared with methylene chloride. The data suggest that the method for media preparation is reproducible.

\section{CONCLUSION}

A simplified methodology for the preparation of FaSSIF and FeSSIF without the use of toxic methylene chloride was studied. The performance of the dissolution media was checked by comparative dissolution of two drug products in FaSSIF and FeSSIF (with and without methylene chloride). 


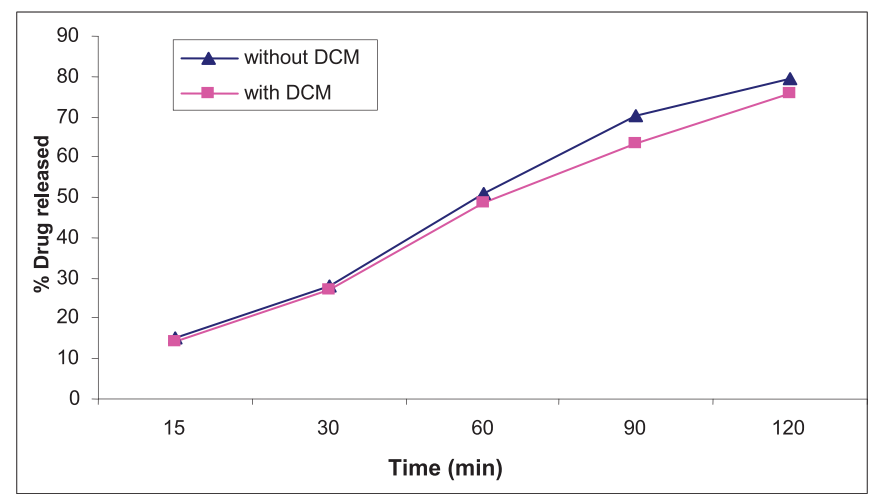

Figure 4. Mean dissolution profiles of metaxalone tablets in FeSSIF media prepared with and without methylene chloride (DCM).

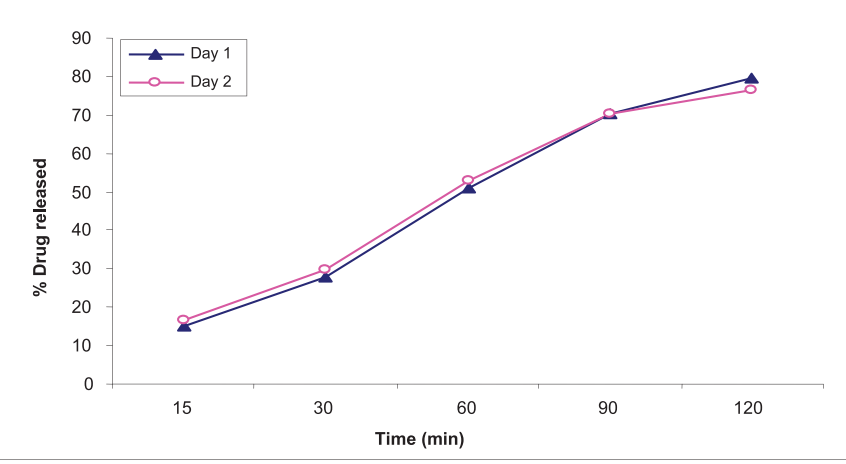

Figure 5. Mean dissolution profiles of metaxalone tablets in FeSSIF media prepared without methylene chloride (DCM) on different days.

The proposed method for the preparation of FaSSIF and FeSSIF without methylene chloride is precise, reproducible, environmentally friendly, and less time consuming.

\section{REFERENCES}

1. Galia, E.; Nicolaides, E.; Hörter, D.; Löbenberg, R.; Reppas, C.; Dressman, J. B. Evaluation of Various

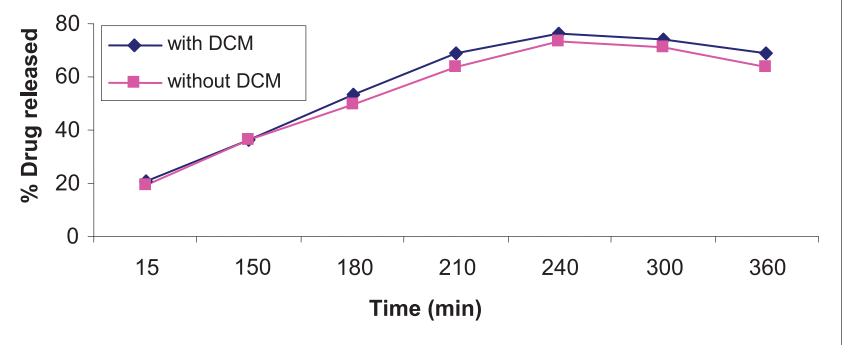

Figure 6. Mean dissolution profiles of dexlansoprazole capsules in FaSSIF media prepared without methylene chloride (DCM) on different days.

Dissolution Media for Predicting In Vivo Performance of Class I and II Drugs. Pharm. Res. 1998, 15 (5), 698705. DOI: 10.1023/A:1011910801212.

2. Kuentz, M. Drug Absorption Modeling as a Tool to Define the Strategy in Clinical Formulation Development. AAPS J. 2008, 10 (3), 473-479. DOI: 10.1208/s12248-008-9054-3.

3. Marques, M. Dissolution Media Simulating Fasted and Fed States. Dissolution Technol. 2004, 11 (2), 16.

4. Ilardia-Arana, D.; Kristensen, H. G.; Müllertz, A. Biorelevant dissolution media: Aggregation of amphiphiles and solubility of estradiol. J. Pharm. Sci. 2006, 95 (2), 248-255. DOI: 10.1002/jps.20494.

5. Boni, J. E.; Brickl, R. S.; Dressman, J.; Pfefferle, M. L. Instant FaSSIF and FeSSIF-Biorelevance Meets Practicality. Dissolution Technol. 2009, 16 (3), 41-45.

6. Kloefer, B.; van Hoogevest, P.; Moloney, R.; Kuentz, M.; Leigh, M. L. S.; Dressman, J. Study of a Standardized Taurochloate-Lecithin Powder for Preparing the Biorelevant Media FeSSIF and FaSSIF. Dissolution Technol. 2010, 17 (3), 6-13. 